TRANSACTIONS OF THE

AMERICAN MATHEMATICAL SOCIETY

Volume 353, Number 4, Pages 1691-1703

S 0002-9947(01)02733-7

Article electronically published on January 2, 2001

\title{
ON COMPOSITE FORMAL POWER SERIES
}

\author{
JACQUES CHAUMAT AND ANNE-MARIE CHOLLET
}

\begin{abstract}
Let $F$ be a holomorphic map from $\mathbb{C}^{n}$ to $\mathbb{C}^{n}$ defined in a neighborhood of 0 such that $F(0)=0$. If the Jacobian determinant of $F$ is not identically zero, P. M. Eakin et G. A. Harris proved the following result: any formal power series such that $\mathcal{A} \circ F$ is analytic is itself analytic. If the Jacobian determinant of $F$ is identically zero, they proved that the previous conclusion is no more true.

The authors get similar results in the case of formal power series satifying growth conditions, of Gevrey type for instance. Moreover, the proofs here give, in the analytic case, a control of the radius of convergence of $\mathcal{A}$ by the radius of convergence of $\mathcal{A} \circ F$.

RÉsumé. Soit $F$ une application holomorphe de $\mathbb{C}^{n}$ dans $\mathbb{C}^{n}$ définie dans un voisinage de 0 et vérifiant $F(0)=0$. Si le jacobien de $F$ n'est pas identiquement nul au voisinage de 0, P.M. Eakin et G.A. Harris ont établi le résultat suivant: toute série formelle $\mathcal{A}$ telle que $\mathcal{A} \circ F$ est analytique est elle-même analytique. Si le jacobien de $F$ est identiquement nul, ils montrent que la conclusion précédente est fausse.

Les auteurs obtiennent des résultats analogues pour les séries formelles à croissance contrôlée, du type Gevrey par exemple. De plus, les preuves données ici permettent, dans le cas analytique, un contrôle du rayon de convergence de $\mathcal{A}$ par celui de $\mathcal{A} \circ F$.
\end{abstract}

\section{INTRODUCTION}

We denote by $\mathcal{F}_{n}$ the ring of formal power series in $n$ variables. Let $\mathcal{A} \in \mathcal{F}_{n}$; we write

$$
\mathcal{A}=\sum_{J \in \mathbb{N}^{n}} a_{J} x^{J}=\sum_{j \in \mathbb{N}} \sum_{J \in \mathbb{N}^{n} ;|J|=j} a_{J} x^{J}=\sum_{j \in \mathbb{N}} H_{j} \mathcal{A}(x),
$$

where $|J|$ denotes the length of the multi-index $J ; H_{j} \mathcal{A}(x)$ is the homogeneous polynomial of degree $j$ in the expansion of $\mathcal{A}$. We shall use the same notation $H_{j} \Phi(x)$ in the power series expansion of a holomorphic function $\Phi$ in a neighborhood of 0 .

Let $\operatorname{ord}(\mathcal{A})=\inf \left(j \in \mathbb{N} ; H_{j} \mathcal{A}(x) \not \equiv 0\right)$ be the vanishing order of $\mathcal{A}$, with the convention $\operatorname{ord}(\mathcal{A})=\infty$ if $\mathcal{A}$ is identically 0 . The same notation will also be used for the vanishing order of a holomorphic function in a neighborhood of 0.

Let $F$ be a holomorphic map from $\mathbb{C}^{n}$ to $\mathbb{C}^{n}$ defined in a ball $B\left(0, r_{0}\right)$ of $\mathbb{C}^{n}$ and such that $F(0)=0$. We denote by $\Phi$ the Jacobian determinant of $F$.

Received by the editors December 12, 1997.

2000 Mathematics Subject Classification. Primary 13F25, 13J05, 32A05.

(C)2001 American Mathematical Society 
Theorem A [2, 4], 7]. The following properties are equivalent:

$$
\operatorname{ord}(\Phi)<\infty
$$

there exist constants $D_{1}$ and $D_{2}$ such that

$$
\text { for any } \mathcal{A} \in \mathcal{F}_{n}, \operatorname{ord}(\mathcal{A} \circ F) \leq D_{1} \operatorname{ord}(\mathcal{A})+D_{2} \text {. }
$$

$D_{1}$ and $D_{2}$ depend only on $F$.

Condition (A.1) implies (A.2) is a step in the proof of the Glaeser Theorem on Composite Functions [4, 7]. We can deduce from it the estimate $D_{1} \leq 2 \operatorname{ord}(\Phi)-1$. The converse is proved by P. M. Eakin and G. A. Harris in (2], lemma 4.2, inequality 4.2.2).

We denote by $\mathcal{O}(n)$ the ring of convergent power series in $n$ variables.

Theorem B 2]. The following properties are equivalent:

$$
\operatorname{ord}(\Phi)<\infty
$$

$$
\text { for any } \mathcal{A} \in \mathcal{F}_{n}, \mathcal{A} \circ F \in \mathcal{O}(n) \text { implies } \mathcal{A} \in \mathcal{O}(n) \text {. }
$$

As mentioned by P. M. Eakin and G. A. Harris, this result can be seen as a corollary of a more general result of A. M. Gabrielov 3 .

In this work, the authors deal with formal power series whose coefficients satisfy growth conditions. More precisely, let $M=\left\{M_{p}\right\}_{p \geq 0}$ be a sequence of real strictly positive numbers satisfying the following properties:

$$
\begin{array}{ll}
\left(H_{1}\right) & M_{0}=1 \text { and }\left\{M_{p}\right\}_{p \geq 0} \text { is logarithmically convex, } \\
\left(H_{2}\right) & M_{p}^{1 / p} \text { tends to infinity with } p .
\end{array}
$$

Let $C$ be a positive constant and, for any $\mathcal{A}$ of $\mathcal{F}(n)$, let

$$
\|\mathcal{A}\|_{C, M}=\sup _{J \in \mathbb{N}^{n}} \frac{\left|a_{J}\right|}{C^{j} M_{j}} .
$$

Here $j$ denotes the length of $J$.

Let $d$ be a strictly positive integer. We denote by $M^{(d)}$ the sequence defined by

$$
M_{n}^{(d)}=M_{d n} \text {, for any integer } n .
$$

Theorem C. Let $F$ be a holomorphic map from $\mathbb{C}^{n}$ to $\mathbb{C}^{n}$ defined in a neigborhood of 0 and let $\Phi$ be its Jacobian determinant. Let $M$ be a sequence of strictly positive real numbers satisfying $\left(H_{1}\right)$ and $\left(H_{2}\right)$. Let $C$ be a strictly positive constant. The following properties are equivalent:

$$
\operatorname{ord}(\Phi)<\infty
$$

there exist $D>0$ and an integer $d \geq 1$ such that for any $\mathcal{A} \in \mathcal{F}_{n},\|\mathcal{A}\|_{D, M^{(d)}} \leq D\|\mathcal{A} \circ F\|_{C, M}$.

The techniques developed here allow us to get precise norm estimates. The parameter $d$ which occurs in (C.2) is optimal, as shown in Remark 18 (a). Moreover, we also get the best constants one should expect in (A.2), as shown in Theorem 17 .

We can also observe that the assumptions on the sequence $M$ are extremely weak. If $M_{p}=p !^{\alpha}, \alpha>0$, we get the classical Gevrey growth condition. It can be verified that the method developed here can be adapted if the sequence $M_{p}$ is identically equal to 1 . This gives a new proof that (B.1) implies (B.2); as in [8], 
we then get a control of the radius of convergence of $\mathcal{A}$ in terms of the radius of convergence of $\mathcal{A} \circ F$.

1. Let $F$ be a holomorphic map from $\mathbb{C}^{n}$ to $\mathbb{C}^{n}$ defined in a ball $B\left(0, r_{0}\right)$ in $\mathbb{C}^{n}$ such that $F(0)=0$. Let $g$ be a holomorphic function in a neighborhood of 0 . For any multi-index $L$ in $\mathbb{N}^{n}$ of length $\ell \geq 1$, we have in a neighborhood of 0

$$
\frac{\partial^{\ell}}{\partial u^{L}}(g \circ F)(x)=\sum_{K \in \mathbb{N}^{n} ; 1 \leq|K| \leq \ell} A_{K, L}(x) \frac{\partial^{k}}{\partial z^{K}}(g) \circ F(x)
$$

with $A_{K, L}$ holomorphic functions in $B\left(0, r_{0}\right)$.

Of course, this formula remains true if $g$ is a formal power series. Moreover, we have the following lemma, which can be proved by induction as in 6, proposition $2.5]$.

Lemma. There exists a constant $C_{1}>0$, depending only on $F$, such that, for all multi-indices $L, K$ and $S$ and any $x$ in $B\left(0, \frac{r_{0}}{2}\right)$,

$$
\left|\frac{\partial^{s}}{\partial x^{S}} A_{K, L}(x)\right| \leq C_{1}^{\ell+s+1}(\ell+s-k) ! .
$$

Here, $k, \ell$ and $s$ denote the lengths of $K, L$ and $S$.

Hence, we deduce immediately that there exists a constant $C_{2}>0$, depending only on $F$, such that, for any $\mathcal{A} \in \mathcal{F}_{n}$ and any $C>0$,

$$
\|\mathcal{A} \circ F\|_{C_{2} C, M} \leq C_{2}\|\mathcal{A}\|_{C, M} .
$$

Our purpose here is to obtain a converse. In fact, we want to discuss the following question:

$$
\text { If, for some } C,\|\mathcal{A} \circ F\|_{C, M} \text { is finite, what can be said of } \mathcal{A} \text { ? }
$$

Inequality (1.3) gives an evident answer if $F$ is a biholomorphic map in a neighborhood of 0 .

In what follows we assume that the Jacobian determinant $\Phi$ of $F$ satisfies

$$
\Phi(0)=0 \quad \text { and } \quad \operatorname{ord}(\Phi)<\infty .
$$

Write

$$
\Phi(x)=\sum_{j \in \mathbb{N}} \sum_{J \in \mathbb{N}^{n} ;|J|=j} \varphi_{J} x^{J}=\sum_{j \in \mathbb{N}} H_{j} \Phi(x)
$$

and $\mu=\operatorname{ord}(\Phi)$; we get then

$$
\begin{aligned}
& H_{j} \Phi(x) \equiv 0 \quad \text { if } j<\mu, \\
& H_{\mu} \Phi(x) \not \equiv 0 .
\end{aligned}
$$

Let

$$
\Sigma=\Phi^{-1}(0) .
$$

2. Geometrical Lemma. There exists an open subset $\Omega_{1}$ of the unit sphere in $\mathbb{C}^{n}$ such that:

$$
z e^{i \theta} \in \Omega_{1} \text {, if } z \text { belongs to } \Omega_{1} \text { and } \theta \text { to }[0,2 \pi] \text {. }
$$

There exists $C_{3}>0$ such that, for any $z$ of $\Omega_{1}$,

$$
\left|H_{\mu} \Phi(z)\right| \geq C_{3} .
$$


There exists $r_{1}>0$ such that, for any $z$ in $\Omega_{1}$ and any $\zeta$ in $\mathbb{C},|\zeta|<r_{1}$,

$$
|\Phi(\zeta z)| \geq \frac{C_{3}}{2}|\zeta|^{\mu} \text {. }
$$

There exists $C_{4}>0$ such that, for any $z$ in $\Omega_{1}$ and any $\zeta$ in $\mathbb{C},|\zeta|<r_{1}$,

$$
C_{4}|\zeta| \leq \operatorname{dist}(\zeta z, \Sigma) \leq|\zeta| \text {. }
$$

Proof. This is a simple consequence of the definition of $\mu$ and of the holomorphy of $\Phi$.

This open subset $\Omega_{1}$ will play an important role in the definition of a new family of norms on $\mathcal{F}_{n}$ (see 7.1).

3. Definitions and Notations. Let $\Gamma_{1}$ be the cone defined by

$$
\Gamma_{1}=\left\{\zeta z ; z \in \Omega_{1}, \zeta \in \mathbb{C},|\zeta|<r_{1}\right\} .
$$

Clearly, $\Gamma_{1} \cap \Sigma=\{0\}$.

Let $x=\left\{x_{1}, \cdots, x_{n}\right\}$ be a point of $B\left(0, r_{1}\right) \backslash \Sigma$ and $g$ a holomorphic function in a neighborhood of $F(x)$. We have

$$
\frac{\partial}{\partial z_{p}}(g \circ F)(x)=\sum_{q=1}^{n} \frac{\partial}{\partial u_{q}}(g) \circ F(x) \frac{\partial}{\partial z_{p}}\left(F_{q}\right)(x), \quad 1 \leq p \leq n .
$$

As, by hypothesis, the Jacobian matrix $J F$ of $F$ is invertible at the point $x$, we get

$$
\frac{\partial}{\partial u_{q}}(g) \circ F(x)=\frac{1}{\Phi(x)} \sum_{p=1}^{n} T_{p, q}(x) \frac{\partial}{\partial z_{p}}(g \circ F)(x), \quad 1 \leq q \leq n .
$$

The functions $T_{p, q}, 1 \leq p \leq n, 1 \leq q \leq n$, are holomorphic in the ball $B\left(0, r_{1}\right)$. Let $\nu$ be the minimum of vanishing orders of the functions $T_{p, q}$. It is the vanishing order of the matrix of cofactors of $J F(x)$. Then, there exists a constant $C_{5}>0$ such that, for any $p, 1 \leq p \leq n$, any $q, 1 \leq q \leq n$, and any $x$ in $B\left(0, r_{1}\right)$,

$$
\left|T_{p, q}(x)\right| \leq C_{5}|x|^{\nu} \text {. }
$$

It follows from (1.4) that, for any $x$ in $B\left(0, r_{1}\right)$, with a suitable constant $C_{6}$, we have

$$
|\Phi(x)| \leq C_{6}|x|^{\mu} \text { and hence } \mu \geq \nu \text {. }
$$

Observe that

$$
\mu>\nu
$$

Indeed, for any $z$ in $\Omega_{1}$ and any $\zeta$ such that $|\zeta|<r_{1}$, we get, from (2.3) and (3.5), $\frac{C_{3}}{2}|\zeta|^{\mu} \leq \mid \Phi\left(\left.\zeta z\left|\leq C_{6}\right| \zeta\right|^{\mu}\right.$. If $\mu=\nu$, it follows that $J F^{-1}(\zeta z)$ is bounded. Then, letting $\zeta$ tend to 0 , we get that $J F(0)$ is invertible. This contradicts (1.4).

Replace $g$ in (3.3) by $\frac{\partial}{\partial u_{m}}(g), 1 \leq m \leq n$. We get then an expression of the derivatives of order 2 of $g$ evaluated at $F(x)$ in terms of the derivatives of order $\leq 2$ of $g \circ F$ evaluated at $x$. By iterating the process, we get, for any multi-index $L$ of $\mathbb{N}^{n}$ of length $\ell \geq 1$ and any $x$ in $B\left(0, r_{1}\right) \backslash \Sigma$,

$$
\frac{\partial^{\ell}}{\partial u^{L}}(g) \circ F(x)=\frac{1}{\Phi(x)^{2 \ell-1}} \sum_{K \in \mathbb{N}^{n} ; 1 \leq|K| \leq \ell} T_{K, L}(x) \frac{\partial^{k}}{\partial z^{K}}(g \circ F)(x)
$$

with $T_{K, L}$ holomorphic functions in $B\left(0, r_{1}\right)$. 
4. Lemma. For any $z$ in $\Gamma_{1}$ and any $\zeta$ in $\mathbb{C},|\zeta| \leq r_{1}$, the functions $T_{K, L}$ appearing in (3.7) satisfy the following properties:

$$
\begin{aligned}
& T_{K, L}(z \zeta)=\zeta^{k-\mu+\ell(\mu+\nu-1)} \sum_{j=0}^{\infty} C_{K, L, j}(z) \zeta^{j}, \\
& \left|C_{K, L, j}(z)\right| \leq r_{1}^{-j} C_{7}^{\ell+1}(\ell-k) ! .
\end{aligned}
$$

Proof. Of course, $k-\mu+\ell(\mu+\nu-1) \geq 0$.

We use (2.3) and (3.4) and we apply proposition 2.5 of [6] with $\omega(t)=t^{\mu-\nu+1}$ and $E=\Gamma_{1}$. Hence, there exists (after shrinking $r_{1}$ and $\Omega_{1}$ if necessary) a constant $C_{8} \geq 1$ such that, for any $z$ in $\Omega_{1}$ and any $\zeta, 0<|\zeta|<r_{1}$,

$$
\left|\frac{T_{K, L}}{\Phi^{2 \ell-1}}(\zeta z)\right| \leq C_{8}^{\ell+1}(\ell-k) !|\zeta|^{k-\ell(\mu-\nu+1)} .
$$

From (4.2) and (3.5), for any $z$ in $\Omega_{1}$ and any $\zeta, 0<|\zeta|<r_{1}$, we have

$$
\left|T_{K, L}(\zeta z)\right| \leq C_{9}^{\ell+1}(\ell-k) !|\zeta|^{k-\mu+\ell(\mu+\nu-1)} .
$$

with $C_{9}$ a suitable constant.

Let $D\left(0, r_{1}\right)$ denote the disk of center 0 and radius $r_{1}$. It follows from (4.3) that, for any given $z$ in $\Omega_{1}$, the function

$$
\zeta \rightarrow \frac{T_{K, L}(\zeta z)}{\zeta^{k-\mu+\ell(\mu+\nu-1)}}
$$

is holomorphic and bounded in $D\left(0, r_{1}\right) \backslash\{0\}$. It extends to a function holomorphic in $D\left(0, r_{1}\right)$ and bounded by $C_{9}^{k+1}(\ell-k)$ !. It has a power series expansion in $D\left(0, r_{1}\right)$; this proves (4.1) by using Cauchy inequalities.

\section{Lemma.}

$$
\begin{gathered}
H_{j} T_{K, L} \equiv 0 \text { if } j<k-\mu+\ell(\mu+\nu-1) \\
\text { and, for any } z \text { in } \Omega_{1} \\
\left|H_{j} T_{K, L}(z)\right| \leq C_{10}^{\ell+1}(\ell-k) ! r_{1}^{k-\mu+\ell(\mu+\nu-1)-j} \text { if } j \geq k-\mu+\ell(\mu+\nu-1)
\end{gathered}
$$

with $C_{10}$ a suitable constant.

Proof. This is just an other way of stating lemma 4 .

6. Remark. From (3.7) we have, for any $x$ in $B\left(0, r_{1}\right)$ and any multi-index $L$ in $\mathbb{N}^{n}$ of length $\ell$,

$$
\Phi(x)^{2 \ell-1} \frac{\partial^{\ell}}{\partial u^{L}}(g) \circ F(x)=\sum_{K \in \mathbb{N}^{n} ;|K| \leq \ell} T_{K, L}(x) \frac{\partial^{k}}{\partial z^{K}}(g \circ F)(x) .
$$

This formula still makes sense if we replace $g$ by a formal power series $\mathcal{A}$. We have the equality $(6.2)$ in the ring $\mathcal{F}_{n}$ :

$$
\Phi^{2 \ell-1} \frac{\partial^{\ell}}{\partial u^{L}}(\mathcal{A}) \circ F=\sum_{K \in \mathbb{N}^{n} ;|K| \leq \ell} T_{K, L} \frac{\partial^{k}}{\partial z^{K}}(\mathcal{A} \circ F) .
$$

7. Definition. We introduce now a new family of norms on $\mathcal{F}_{n}$ well adapted to $\Omega_{1}$ and to the estimates obtained in lemma 5. Denote, for any $\mathcal{A}$ in $\mathcal{F}_{n}$,

$$
\|\mathcal{A}\|_{C, M, \Omega_{1}}=\sup _{j \in \mathbb{N}} \frac{\sup _{z \in \Omega_{1}}\left|H_{j} \mathcal{A}(x)\right|}{C^{j} M_{j}} .
$$


8. Key Lemma. There exist two strictly positive constants $C_{11}$ and $C_{12}$, depending only on $\Omega_{1}$ such that, for any $\mathcal{A}$ in $\mathcal{F}_{n}$ and any $C>0$,

$$
\|\mathcal{A}\|_{C, M} \leq\|\mathcal{A}\|_{C_{11} C, M, \Omega_{1}} \leq\|\mathcal{A}\|_{C_{12} C, M}
$$

We have, for any $\mathcal{A}$ in $\mathcal{F}_{n}$, any constant $C$ and any multi-index $K$ of length $k$,

$$
\sup _{|x|=1}\left|H_{j} \frac{\partial^{k}}{\partial z^{K}}(\mathcal{A})(x)\right| \leq k !(2 n C)^{j+k} M_{j+k}\|\mathcal{A}\|_{C, M} .
$$

Proof. It is known from [1] that there exists a constant $C_{13}>0$ such that, for any homogeneous polynomial of degree $j$ in the variable $x$ in $\mathbb{C}^{n}$, we have

$$
C_{13}^{j} \sup _{J \in \mathbb{N}^{n}}\left|p_{J}\right| \leq \sup _{|x|=1}\left|P_{j}(x)\right| \leq n^{j} \sup _{J \in \mathbb{N}^{n}}\left|p_{J}\right|
$$

Here we write $P_{j}(x)=\sum_{J \in \mathbb{N}^{n} ;|J|=j} p_{J} x^{J}$.

It is known also from [5] that, if $\Omega_{1}$ is an open subset of the unit sphere in $\mathbb{C}^{n}$, there exists a constant $C_{14}\left(\Omega_{1}\right)$ such that, for any homogeneous polynomial $P_{j}$ of degree $j$, we have

$$
\sup _{|x|=1}\left|P_{j}(x)\right| \leq\left(C_{14}\left(\Omega_{1}\right)\right)^{j} \sup _{x \in \Omega_{1}}\left|P_{j}(x)\right| .
$$

From (8.3) and (8.4), we get (8.1).

In order to prove $(8.2)$, we write

$$
\begin{aligned}
& \frac{\partial^{k}}{\partial z^{K}}(\mathcal{A})=\sum_{T \geq K} a_{T} \frac{\partial^{k}}{\partial z^{K}}\left(x^{T}\right)=\sum_{T \geq K} a_{T} C_{T, K} x^{T-K} \\
& \text { with }\left|C_{T, K}\right| \leq k ! 2^{|T|} .
\end{aligned}
$$

Inequality (8.2) is an immediate consequence of (8.5) and (8.3).

9. Definition. To the sequence $M=\left\{M_{p}\right\}_{p \geq 0}$, we associate the function $h_{M}$ defined by

$$
h_{M}(r)=\inf _{p \geq 0} r^{p} M_{p}, r>0 .
$$

From $\left(H_{2}\right)$, we claim that $h_{M}$ is a strictly positive increasing function.

10. Lemma. For any $\mathcal{A}$ in $\mathcal{F}_{n}$ and any multi-index $L$ in $\mathbb{N}^{n}$ of length $\ell$, put

$$
\mathcal{S}_{L}=\sum_{K \in \mathbb{N}^{n} ;|K| \leq \ell} T_{K, L} \frac{\partial^{k}}{\partial z^{K}}(\mathcal{A} \circ F) .
$$

Then

$$
\begin{aligned}
& H_{j} \mathcal{S}_{L} \equiv 0 \text { for } j \leq \ell(\mu+\nu-1)-\mu \text { and } \\
& \begin{aligned}
& \sup _{z \in \Omega_{1}} \mid H_{j} \mathcal{S}_{L}(z) \mid \\
& \leq h_{M}^{-1}\left(C_{15} C\right)\|\mathcal{A} \circ F\|_{C, M} \ell ! C_{16}^{\ell+1}\left(C_{16} C\right)^{j+\mu-\ell(\mu+\nu-1)} M_{j+\mu-\ell(\mu+\nu-1)} \\
& \quad \text { if } j>\ell(\mu+\nu-1)-\mu .
\end{aligned}
\end{aligned}
$$

Here, $C_{15}$ and $C_{16}$ are suitable strictly positive constants depending only on $F$. 
Proof. Write, for simplicity,

$$
\mathcal{B}_{K}=\frac{\partial^{k}}{\partial z^{K}}(\mathcal{A} \circ F)=\sum_{j} H_{j} \mathcal{B}_{K}(x) .
$$

We get

$$
T_{K, L} \frac{\partial^{k}}{\partial z^{K}}(\mathcal{A} \circ F)=\sum_{m \in \mathbb{N}} \sum_{q+j=m} H_{q} T_{K, L}(x) H_{j} \mathcal{B}_{K}(x) .
$$

Using then (5.1), (7.1) and (8.2), we get

$$
\begin{aligned}
& H_{m}\left(T_{K, L} \mathcal{B}_{K}\right) \equiv 0, \text { for } m<k-\mu+\ell(\mu+\nu-1) \\
& \text { and, for } m \geq k-\mu+\ell(\mu+\nu-1) \text { and } z \text { in } \Omega_{1}, \\
& \left|H_{m}\left(T_{K, L} \mathcal{B}_{K}\right)\right| \leq \sum_{q+j=m ; q \geq k-\mu+\ell(\mu+\nu-1)} C_{10}^{\ell+1}(\ell-k) ! r_{1}^{k-\mu+\ell(\mu+\nu-1)-q} \\
& \leq \ell ! C_{10}^{\ell+1}\|\mathcal{A} \circ F\|_{C, M} \sum_{q+j=m ; q \geq k-\mu+\ell(\mu+\nu-1)} r_{1}^{k-\mu+\ell(\mu+\nu-1)-q}(2 n C)^{j+k} M_{j+k} k !\|\mathcal{A} \circ F\|_{C, M}
\end{aligned}
$$

Observe now, from definition (9.1), that, for any $s \in \mathbb{N}, s>0$,

$$
r_{1}^{-s} \leq(n C)^{s} M_{s} h_{M}^{-1}\left(r_{1} n C\right) .
$$

We deduce from that, using $\left(H_{1}\right)$,

$$
\begin{aligned}
& \quad \sum_{q+j=m ; q \geq k-\mu+\ell(\mu+\nu-1)} r_{1}^{-[q-(k-\mu+\ell(\mu+\nu-1))]}(2 n C)^{j+k} M_{j+k} \\
& \leq \sum_{q+j=m ; q \geq k-\mu+\ell(\mu+\nu-1)}(n C)^{q-(k-\mu+\ell(\mu+\nu-1))} M_{q-(k-\mu+\ell(\mu+\nu-1))} \\
& \times h_{M}^{-1}\left(r_{1} n C\right)(2 n C)^{j+k} M_{j+k} \\
& \leq h_{M}^{-1}\left(r_{1} n C\right)(2 n C)^{m+\mu-\ell(\mu+\nu-1)} M_{m+\mu-\ell(\mu+\nu-1)} \sum_{t=0}^{\infty}\left(\frac{1}{2}\right)^{t} \\
& \leq 2 h_{M}^{-1}\left(r_{1} n C\right)(2 n C)^{m+\mu-\ell(\mu+\nu-1)} M_{m+\mu-\ell(\mu+\nu-1)} .
\end{aligned}
$$

This gives the anounced result, because the number of terms of the sum $\sum_{|K| \leq \ell}$ in (10.1) is bounded by $n^{\ell}$.

11. Lemma. Let $\mathcal{B}$ belonging to $\mathcal{F}_{n}$ and $\alpha$ an integer, $\alpha>0$. Write

$$
\Phi^{\alpha} \mathcal{B}=\sum_{j \in \mathbb{N}} H_{j}\left(\Phi^{\alpha} \mathcal{B}\right)(x)
$$

and suppose that there exist $\beta \geq-1, C_{17}$ and $C_{18}$ such that

$$
\begin{aligned}
& H_{j}\left(\Phi^{\alpha} \mathcal{B}\right) \equiv 0 \text { for } j \leq \beta \leq \mu \alpha \text { and } \\
& \sup _{z \in \Omega_{1}}\left|H_{j}\left(\Phi^{\alpha} \mathcal{B}\right)(z)\right| \leq C_{17} C_{18}^{j-\beta} M_{j-\beta} \text { if } j>\beta .
\end{aligned}
$$


Then, we have

$$
\begin{gathered}
\mathcal{B}=\sum_{j \in \mathbb{N}} H_{j} \mathcal{B}(x) \text { with } \\
\sup _{z \in \Omega_{1}}\left|H_{j} \mathcal{B}(z)\right| \leq 2 C_{17}\left(\frac{C_{3}}{2}\right)^{-\alpha} h^{-1}\left(\frac{C_{18} r_{1}}{2}\right)\left(C_{18}\right)^{\mu \alpha-\beta+j} M_{\mu \alpha-\beta+j} .
\end{gathered}
$$

Proof. For $z$ in $\Omega_{1}$ and $\zeta$ in $\mathbb{C},|\zeta| \leq r_{1}$, we can write

$$
\Phi(\zeta z)=\zeta^{\mu} \Psi(\zeta, z) .
$$

Then, from (2.3), we get

$$
|\Psi(\zeta, z)| \geq \frac{C_{3}}{2}
$$

Let

$$
\Psi^{-\alpha}(\zeta, z)=\sum_{j=0}^{\infty} \psi_{j}(\alpha, z) \zeta^{j}
$$

It follows from the Cauchy formulas that

$$
\left|\psi_{j}(\alpha, z)\right| \leq\left(\frac{C_{3}}{2}\right)^{-\alpha} r_{1}^{-j}
$$

We get then, for $z$ in $\Omega_{1}$,

$$
\Phi^{\alpha}(\zeta z) \mathcal{B}(\zeta z)=\sum_{j \in \mathbb{N}} H_{j}\left(\Phi^{\alpha} \mathcal{B}\right)(z) \zeta^{j}=\zeta^{\mu \alpha} \Psi^{\alpha}(\zeta, z) \sum_{j \in \mathbb{N}} H_{j} \mathcal{B}(z) \zeta^{j}
$$

and

$$
\begin{aligned}
\zeta^{\mu \alpha} \sum_{j \in \mathbb{N}} H_{j} \mathcal{B}(z) \zeta^{j} & =\sum_{j=0}^{\infty} \psi_{j}(\alpha, z) \zeta^{j} \sum_{j \geq \beta} H_{j}\left(\Phi^{\alpha} \mathcal{B}\right)(z) \zeta^{j} \\
& =\sum_{s \in \mathbb{N}} \zeta^{s} \sum_{t+j=s} \psi_{t}(\alpha, z) H_{j}\left(\Phi^{\alpha} \mathcal{B}\right)(z)
\end{aligned}
$$

We have

$$
\begin{gathered}
\sum_{t+j=s} \psi_{t}(\alpha, z) H_{j}\left(\Phi^{\alpha} \mathcal{B}\right)(z) \equiv 0 \text { for } s \leq \beta, \text { and } \\
\left|\sum_{t+j=s} \psi_{t}(\alpha, z) H_{j}\left(\Phi^{\alpha} \mathcal{B}\right)(z)\right| \leq \sum_{j+t=s}\left(\frac{C_{3}}{2}\right)^{-\alpha} r_{1}^{-t} C_{17} C_{18}^{j-\beta} M_{j-\beta}
\end{gathered}
$$

As in the proof of lemma 10, we remark that

$$
r_{1}^{-t} \leq\left(\frac{C_{18}}{2}\right)^{t} M_{t} h_{M}^{-1}\left(\frac{r_{1} C_{18}}{2}\right)
$$


and hence we get

$$
\begin{aligned}
& \left|\sum_{t+j=s} \psi_{t}(\alpha, z) H_{j}\left(\Phi^{\alpha} \mathcal{B}\right)(z)\right| \\
& \quad \leq C_{17}\left(\frac{C_{3}}{2}\right)^{-\alpha} h_{M}^{-1}\left(\frac{r_{1} C_{18}}{2}\right) \sum_{j+t=s} 2^{-t} C_{18}^{t+j-\beta} M_{t} M_{j-\beta} \\
& \leq 2 C_{17}\left(\frac{C_{3}}{2}\right)^{-\alpha} h_{M}^{-1}\left(\frac{r_{1} C_{18}}{2}\right) C_{18}^{s-\beta} M_{s-\beta} .
\end{aligned}
$$

We have

$$
H_{i} \mathcal{B}(z)=\sum_{t+j=i+\mu \alpha} \psi_{t}(\alpha, z) H_{j}\left(\Phi^{\alpha} \mathcal{B}\right)(z)
$$

and hence

$$
\left|H_{i} \mathcal{B}(z)\right| \leq 2 C_{17}\left(\frac{C_{3}}{2}\right)^{-\alpha} h_{M}^{-1}\left(\frac{r_{1} C_{18}}{2}\right) C_{18}^{i+\mu \alpha-\beta} M_{i+\mu \alpha-\beta} .
$$

Let $d$ be a strictly positive integer. We denote by $M^{(d)}$ the sequence defined by $M_{n}^{(d)}=M_{d n}$ for any $n$.

12. Proposition. We have, for any $\mathcal{A}$ in $\mathcal{F}_{n}$ and any multi-index $L$ of length $\ell$,

$$
\sup _{|x|=1}\left|H_{j} \frac{\partial^{\ell}}{\partial z^{L}}(\mathcal{A}) \circ F(x)\right| \leq \ell ! C_{19}\left(C_{20} C\right)^{j+(\mu-\nu+1) \ell} M_{j+(\mu-\nu+1) \ell}\|\mathcal{A} \circ F\|_{C, M}
$$

and hence

$$
\|\mathcal{A}\|_{C_{21} C^{\mu-\nu+1}, M^{(\mu-\nu+1)}} \leq C_{22}\|\mathcal{A} \circ F\|_{C, M} .
$$

The constants $C_{20}$ and $C_{21}$ depend only on $F$. The constants $C_{19}$ and $C_{22}$ depend also on $C$.

Proof. Using (6.2) and the notation (10.1), we have

$$
\Phi^{2 \ell-1} \frac{\partial^{\ell}}{\partial u^{L}}(\mathcal{A}) \circ F=\mathcal{S}_{L} .
$$

By (10.2), we have a control of $\sup _{z \in \Omega_{1}}\left|H_{j} \mathcal{S}_{L}(z)\right|$. From lemma 11 applied with $\alpha=2 \ell-1, \beta=\ell(\mu+\nu-1)-\mu$ and $\mathcal{B}=\frac{\partial^{\ell}}{\partial u^{L}}(\mathcal{A}) \circ F$ we get a control of $\sup _{z \in \Omega_{1}}\left|H_{j} \mathcal{B}(z)\right|$.

From there, using (8.1), we deduce (12.1).

The inequality (12.2) is a consequence if we consider for any $L$ the constant term in $\frac{\partial^{\ell}}{\partial u^{L}}(\mathcal{A}) \circ F$. This term is equal to $L ! a_{L}$; we now use the fact that $\frac{\ell !}{L !}$ is bounded by $n^{\ell}$ to conclude.

We show in the next section that the results given here are the best one should expect. We discuss first the case when condition (1.4) is not satisfied.

13. Lemma [2]. Let $F$ be a holomorphic map from $\mathbb{C}^{n}$ to $\mathbb{C}^{n}$ defined in a ball $B\left(0, r_{0}\right)$ in $\mathbb{C}^{n}$ satisfying $F(0)=0$ and whose Jacobian determinant $\Phi$ is identically zero. There exist two holomorphic maps $F_{1}$ and $F_{2}$ from $\mathbb{C}^{n}$ to $\mathbb{C}^{n}$ defined in a neighborhood of 0 satisfying $F_{1}(0)=F_{2}(0)=0$, whose Jacobian determinants are 
not identically zero in a neighborhood of 0 such that $F_{1} \circ F \circ F_{2}$ depends only on the $n-1$ first variables.

Write

$$
G=F_{1} \circ F \circ F_{2} \text {. }
$$

14. Lemma [2. For any large enough integer $\alpha$, there exists a polynomial $P_{\alpha}$ of $n$ variables satisfying the following properties:

$$
\begin{gathered}
P_{\alpha}(z)=\sum_{K, k \leq \alpha} a_{K, \alpha} z^{K}, \\
\sup _{K, k \leq \alpha}\left|a_{K, \alpha}\right|=1, \\
\operatorname{ord}\left(P_{\alpha} \circ G\right) \geq C_{23} \alpha^{n / n-1} .
\end{gathered}
$$

The constant $C_{23}$ depends only on $G$ defined in (13.1).

15. Proposition. Let $F$ be a holomorphic map from $\mathbb{C}^{n}$ to $\mathbb{C}^{n}$ defined in a ball $B\left(0, r_{0}\right)$ in $\mathbb{C}^{n}$ satisfying $F(0)=0$ and whose Jacobian determinant $\Phi$ is identically zero. Let $M$ be a sequence of strictly positive real numbers satisfying $\left(H_{1}\right)$ and $\left(H_{2}\right)$. Let $C$ and $D$ two strictly positive constants and $e$ an integer, $e \geq 1$. There exists a formal power series $\mathcal{A}$ which does not satisfy the inequality

$$
\|\mathcal{A}\|_{D, M(e)} \leq D\|\mathcal{A} \circ F\|_{C, M}<\infty \text {. }
$$

Proof. We use lemmas 13 and 14 and the function $G$ defined in (13.1).

From (14.1) and (14.2), we have

$$
\left\|P_{\alpha}\right\|_{D, M^{(e)}} \geq \frac{1}{D^{\alpha} M_{e \alpha}}, \text { for any } \alpha \text { large enough. }
$$

From (1.1), (1.2) and (14.2), we get, for any multi-index $L$ and any $\alpha$ large enough,

$$
\left|\frac{\partial^{\ell}}{\partial z^{L}}\left(P_{\alpha} \circ G\right)(0)\right| \leq \ell ! C_{24}^{\ell+1} .
$$

The constant $C_{24}$ depends only on $G$.

Denote by $E(\alpha)$ the integer part of $C_{23} \alpha^{n / n-1}+1$. From (14.3) and (15.2), we have, for $\alpha$ large enough,

$$
\left\|P_{\alpha} \circ G\right\|_{C, M} \leq \frac{C_{25}^{E(\alpha)}}{M_{E(\alpha)}} .
$$

The constant $C_{25}$ depends only on $C$ and $G$.

We get from (15.1), (15.3), $\left(H_{2}\right)$ and the previous definition of $E(\alpha)$ that there exists $\alpha$ large enough such that

$$
\left\|P_{\alpha}\right\|_{D, M^{(e)}} \leq D\left\|P_{\alpha} \circ G\right\|_{C, M}
$$

is not fulfilled.

Since, by construction, the maps $F_{1}$ and $F_{2}$ satisfy inequalities (12.2), we get from there the announced result. 
16. Theorem C. Let $F$ be a holomorphic map from $\mathbb{C}^{n}$ to $\mathbb{C}^{n}$ defined in a neighborhood of 0 with Jacobian determinant $\Phi$.

Let $M$ be a sequence of strictly positive real numbers satisfying $\left(H_{1}\right)$ and $\left(H_{2}\right)$. Let $C$ be a strictly positive constant. The two following properties are equivalent:

$$
\operatorname{ord}(\Phi)<\infty
$$

$$
\begin{gathered}
\text { there exist } D, D>0 \text { and } d \in \mathbb{N}, d \geq 1 \text { such that } \\
\text { for any } \mathcal{A} \in \mathcal{F}_{n}, \text { we have }\|\mathcal{A}\|_{D, M^{(d)}} \leq D\|\mathcal{A} \circ F\|_{C, M} .
\end{gathered}
$$

Proof. This is a consequence of propositions 12 and 15.

We can give now a precise version of "(A.1) implies (A.2)".

17. Theorem. Let $F$ be a holomorphic map from $\mathbb{C}^{n}$ to $\mathbb{C}^{n}$ defined in a neigborhood of 0 and let $\Phi$ be its Jacobian determinant. Let $\mu$ denote the vanishing order of $\Phi$ and $\nu$ defined as in section 3.

Suppose that

$$
\operatorname{ord}(\Phi)<\infty
$$

Then

$$
\text { for any } \mathcal{A} \in \mathcal{F}_{n}, \operatorname{ord}(\mathcal{A} \circ F) \leq(\mu-\nu+1) \operatorname{ord}(\mathcal{A}) .
$$

Proof. Denote by $o_{1}$ the vanishing order of $\mathcal{A} \circ F$ and by $o_{2}$ the vanishing order of $\mathcal{A}$.

From definition (10.1)

$$
\mathcal{S}_{L}=\sum_{K \in \mathbb{N}^{n} ;|K| \leq \ell} T_{K, L} \frac{\partial^{k}}{\partial z^{K}}(\mathcal{A} \circ F),
$$

and by (4.1) it is clear that

$$
\operatorname{ord}\left(\mathcal{S}_{L}\right) \geq o_{1}-\mu+\ell(\mu+\nu-1)
$$

On the other hand, there exists a multi-index $P$ of length $p=o_{2}$ such that

$$
\frac{\partial^{p}}{\partial u^{P}}(\mathcal{A}) \neq 0
$$

By (12.3), we get

$$
\operatorname{ord}\left(\mathcal{S}_{P}\right)=\left(2 o_{2}-1\right) \mu \text {. }
$$

Using (17.1) with $L=P$ and (17.2), we have

$$
\left(2 o_{2}-1\right) \mu \geq o_{1}-\mu+o_{2}(\mu+\nu-1) \text {. }
$$

This gives the result.

18. Remarks. (a) The estimate (12.2) is the best possible, as the following example shows.

Example. Let $\sigma_{1}, \cdots, \sigma_{n}$ be the elementary symmetric functions in $n$ variables. Denote by $S$ the map from $\mathbb{C}^{n}$ to $\mathbb{C}^{n}$ defined by

$$
S(x)=\left(\sigma_{1}(x), \sigma_{2}(x), \ldots, \sigma_{n}(x)\right) .
$$

It is easily seen that $\mu=\frac{n(n-1)}{2}$, and $\nu=\frac{(n-1)(n-2)}{2}$ and $\mu-\nu+1=n$. 
If $\mathcal{A}=\sum_{p} a_{p} x_{n}^{p}$ is a formal power series depending only on the last variable $x_{n}$, we have

$$
\|\mathcal{A}\|_{C^{n}, M^{(n)}}=\|\mathcal{A} \circ S\|_{C, M}
$$

Hence, inequality (C.2) is the best one should expect with $d=\mu-\nu+1=n$.

More generally, let $P_{1}, \cdots, P_{n}$ be homogeneous polynomials in $n$ variables of degree respectively $d_{1} \leq \cdots \leq d_{n}$. Denote by $P$ the map from $\mathbb{C}^{n}$ to $\mathbb{C}^{n}$ defined by

$$
P(x)=\left(P_{1}(x), P_{2}(x), \ldots, P_{n}(x)\right) .
$$

If the order of the Jacobian determinant of $P$ is finite, one can easily prove that inequality (C.2) is optimal with $d=d_{n}$.

(b) Under the hypothesis ord $(\Phi)=\infty$, a formal power series $\mathcal{A}$ can be constructed in such a way that, for any constant $D$ and any integer $d$,

$$
\|\mathcal{A}\|_{D, M^{(d)}}=\infty \quad \text { and } \quad\|\mathcal{A} \circ F\|_{1, M}<\infty .
$$

(c) With slight modifications we can adapt the proof of proposition 12 when $M_{p}=1$ for every $p$. Write $M=\mathbb{1}$ in that case. We get hence a new proof of

$$
\text { (B.1) implies (B.2). }
$$

Moreover, if we assume that $C$ has been chosen such that $2 n C \geq \frac{1}{r_{1}}$, we have

$$
\text { for any } \mathcal{A} \in \mathcal{F}_{n},\|\mathcal{A}\|_{E C^{\mu-\nu+1}, \mathbb{1}} \leq E\|\mathcal{A} \circ F\|_{C, \mathbb{1}} \text {. }
$$

This means that if the radius of convergence $\rho_{\mathcal{A} \circ F}$ is small enough, then the radius of convergence $\rho_{\mathcal{A}}$ satisfies the following inequality:

$$
\left(\rho_{\mathcal{A} \circ F}\right)^{\mu-\nu+1} \leq E \rho_{\mathcal{A}}
$$

Here, the radius of convergence of a power series $\mathcal{A}$ is the largest real number $\rho$ such that $\mathcal{A}$ converges in the polydisk of center 0 and radius $\rho$.

The example given in (a) shows that the above inequality is the best possible. On this topic, see also J. C. Tougeron [8].

(d) We have shown that $d=\mu+\nu-1$ is optimal for $S$ in (a). Nevertheless, given a function $F$, finding the best possible integer $d$ in (C.2) and the best constant $D_{1}$ in (A.2) would be of interest.

(e) Theorem $\mathrm{C}$ can be seen as a version of the Glaeser composite functions theorem "above a point" for ultradifferentiable classes ([4], 6]).

\section{REFERENCES}

1. CARTAN H. Calcul Différentiel. Hermann, Paris (1967). MR 36:6243

2. EAKIN P. M. - HARRIS G. A. When $\Phi(f)$ Convergent Implies $f$ is Convergent. Math. Ann. 229 (1977) 201-210. MR 56:3001

3. GABRIELOV A. M. Formal Relations Between Analytic Functions. Math. USSR. Isvestija 7 (1973) 1056-1088. MR 49:10910

4. GLAESER G. Fonctions composées différentiables. Ann. of Math. (2) 77 (1963) 193-209. MR 26:624

5. KLIMEK M. Pluripotential Theory. London Math. Soc. Monographs. Clarendon Press (1991). MR 93h:32021

6. THILLIEZ V. Sur les fonctions composées différentiables. J. Math. Pures et Appl. 76 (1997) 499-524. MR 99e:58018 
7. TOUGERON J. C. Idéaux de fonctions différentiables. Springer Verlag (1972). MR 55:13472

8. TOUGERON J. C. Sur les racines d'un polynôme à coefficients séries formelles. Real Analysis and Algebraic Geometry (Trento, 1988), Lecture Notes in Math., vol.1420 (1990), 325-363. MR 91b:32010

U.M.R. C.N.R.S. 8628, Université Paris-Sud, Mathématiques - BÂt. 425, 91405 Orsay Cedex, France

E-mail address: jacques.chaumat@math.u-psud.fr

U.M.R. C.N.R.S. 8524, Université De Lille, U.F.R. De Mathématique,59655 Villeneuve D'Ascq Cedex, France

E-mail address: chollet@aglae.univ-lille1.fr 